Ekaterina Vladimirovna Kazakova

Institut de la Philologie et du Journalisme, Université nationale de recherche N. Lobachevski, Nijni Novgorod Russie

kazakova.e.v@gmail.com
UDK 821.133.1.09-992:711.432(470)

DOI: $10.4312 /$ vestnik.11.303-313

\title{
L'IMAGE DE MOSCOU DANS LES RÉCITS DE VOYAGE DES ÉCRIVAINS FRANÇAIS CONTEMPORAINS
}

Les récits de voyage, en tant qu'objet d'étude, se situent à l'intersection de plusieurs branches des sciences humaines où priment la littérature comparative, l'imagologie et la psychologie sociale, apportant chacune l'explication du phénomène de l'image de l'Autre (personne, nation, culture, espace). Cette image n'est rarement stéréotypée car ressort, souvent inconsciemment, des représentations bien formées dans l'esprit de celui qui part en voyage pour décrire ensuite le vu et le vécu.

Les récits de voyage qui se pratiquent depuis l'Antiquité, évoluent en fonction de l'époque dans le sens historique, culturel, social, voire international. Avec le temps, les voyages deviennent de moins en moins rares. Et pourtant, le siècle des Lumières ne compte pas encore beaucoup de voyageurs libres (auteurs, artistes, philosophes), ceux qui se déplacent « pour le plaisir », comme on le disait à l'époque, pour les distinguer des voyageurs de profession qui étaient alors plus nombreux (marchands, marins, soldats, missionnaires), mais qui étaient souvent loin de vouloir philosopher sur leur voyages (Moureau 2016 : 66).

On retrouve des récits soit engagés par la « patrie », vu que l'image d'un étranger n'échappe pas aux « pressions politiques, socio-culturelles, ni au rapport de forces [...] entre les cultures » (Pageaux Recherches 136-137), soit conditionnés par le pays hôte. L'étude des récits de Diderot, rendant compte de son voyage jusqu'à la Russie, démontre bien cette conditionnalité (création des villages Potiomkine, les vaines tentatives de l'ambassadeur de la tsarine russe aux Pays-Bas de saisir l'autre variante des réflexions de Diderot sur la visite à Saint-Pétersbourg où ce dernier révèle la réalité entrevue dans la capitale et lors des entretiens en dehors du palais - « Observations sur le Nakaz » - (Moureau 2016 : 77-78).

Ainsi, les récits de voyage, souvent convenus, des littéraires du siècle des Lumières laissent place à des visions et des expressions plus libérées mais encore très souvent contrôlées pour des raisons politiques et sociales différentes. Aujourd'hui, les voyages ne 
sont plus un privilège des fortunés et le voyageur est libre de choisir le milieu qui intéresse son esprit de découvreur et d'autant plus libre de s'exprimer à son propos.

Et pourtant, malgré la liberté totale de s'exprimer, les études comparatives démontrent une certaine unanimité de ces récits contemporains. Mais c'est un autre genre de parti pris qui s'explique par le phénomène de la vision stéréotypée de l'Autre ressortant de la conscience nationale culturelle de celui qui « regarde ». Les stéréotypes, formant une sorte de « forteresse » selon la définition de Walter Lippmann, servent à nous protéger contre l'autre monde culturel et contribue à notre prise de position et à la vision de soi (dans le sens généralisant), de sa nation (Lippmann :1922).

Les stéréotypes ont souvent une mauvaise réputation, souligne Philippe Beck (2010 : 57). Cette remarque est d'autant plus vraie quand on entreprend l'étude des récits de voyage. Et plus le territoire est reculé, plus les points de divergence se multiplient quant à la perception des deux pays (voire des deux cultures) par les auteurs.

Cependant, les nombreux savoirs que l'humain possède sur le monde actuellement (plusieurs stéréotypes en découlent) ainsi que des facteurs d'ordre personnel et national, peuvent conditionner les variations des visions d'un seul territoire par les représentants d'une seule culture - images - ce que la présente étude est censée démontrer.

La Russie est un espace et une culture plutôt exotique pour la conscience européenne. Elle a toujours intéressé voyageurs, écrivains, chercheurs. Avec le développement des moyens de transport et l'accroissement des opportunités financières, augmente le nombre de personnes prêtes à partager leur expérience pratique et émotionnelle avec le lecteur. Dans le même temps, on remarque un changement du côté des exigences de ce dernier, concernant les notes de voyage.

L'intérêt pour la Russie a réuni des écrivains français célèbres tels que Théophile Gautier, Astolphe De Custine, Alexandre Dumas, Jules Verne, Blaise Cendrars. Les deux derniers et, en partie, Dumas, ont créé des exemples de fiction littéraire, qui continuent à servir de points de repères pour les voyageurs et les écrivains du $\mathrm{XX}^{\mathrm{e}}$ siècle. Les touristes suivent les traces de Michel Strogoff, à la recherche constante des illustrations de son œuvre, des coïncidences dans le paysage et des coutumes de l'espace russe, tandis que les écrivains s'inspirent de la "prose » de Cendrars et s'interrogent sur la fiabilité de la révélation de la Russie.

En effet, Blaise Cendrars est devenu une figure emblématique, une sorte de kilomètre zéro, à partir duquel commence un nouveau voyage moderne de la pensée littéraire française à travers l'espace russe. On parlera d'espace, puisque le nom de l'auteur du voyage transsibérien rêvé est choisi pour désigner le train qui part en mai 2010 (Année croisée France-Russie) de Moscou à Vladivostok. Parmi les voyageurs on rencontre des 
écrivains, journalistes et photographes français, ouverts à de nouveaux territoires, de nouvelles rencontres et impressions. Le voyage a abouti à de nombreuses œuvres littéraires et artistiques, émissions de radio et publications dans des périodiques.

Les œuvres créées sous l'impression du voyage sont variées en termes de genre. Celles créées dans le style romanesque conduisent les personnages principaux selon une trajectoire spatiale donnée (Maylis de Kerangal, Mathias Enard), concentrant l'attention du lecteur sur l'évolution dramatique des événements, attribuant au voyage le rôle des décors plus ou moins importants pour l'intrigue. Il y a d'autres travaux : des récits de voyage (travelogues). Les résultats remarquables du voyage sont les travelogues du membre de l'Académie française, Dominique Fernandez (Transsibérien, 2012) et une sorte de rétrospective des visites en Russie d'une voyageuse, Géraldine Dunbar (Seule sur le Transsibérien, 2016).

Pour une analyse plus complète de l'image de Moscou, des fragments métropolitains issus d'autres récits de voyage français ont été examinés. Celui de l'écrivain et professeur d'histoire et de géographie Hervé Bellec (Les Sirènes du Transsibérien, 2008) et celui de l'écrivain et aventurier Sylvain Tesson (Berezina, 2015). Tous les récits sont consacrés au voyage à Vladivostok, en train ou en voiture, à l'exception du livre de Sylvain Tesson qui raconte son trajet à moto (side-car) sur les traces de l'armée de Napoléon en retraite en 1812 .

Tous les récits de voyage débutent dans la ville de Moscou. Même si, pour certains, ce voyage n'est pas le premier, le point de départ de la connaissance émotionnelle et culturelle du territoire est identique et met donc les auteurs dans des conditions égales afin de reconstruire l'image de la capitale russe. Les stéréotypes nationaux font partie intégrante des images de Moscou créées dans les récits de voyage analysés. D'autres composantes servent à rendre ces images complètes, vivantes même : c'est non seulement la perception de l'espace, mais aussi celle des gens, de la météo, du transport, de la mentalité, des contacts éventuels, de l'architecture, de l'attitude de l'Autre envers son espace et son environnement perçue par l'œil attentif de l'écrivain-voyageur. C'est également le choix de l'objet auquel la ville est comparée (animé ou même pas), la composition des textes (dialogues, monologues, mode de description, introduction et présentation des personnages en lien avec l'auteur), le choix du lexique, le penchant pour les lacunes lexicales et culturelles introduites dans les récits dans une certaine mesure et mises en italique tout au long des textes, ce qui rend les images encore plus exotiques. Tout cela contribue à la création de l'image de Moscou par l'auteur. Certains de ces procédés reviennent à des stéréotypes qui, selon Philippe Beck, « font partie d'un arsenal de processus cognitif qui nous permet de gérer la complexité des informations auxquelles nous sommes confrontés quotidiennement » $(2010: 57)$.

Ces morceaux de récits imagologiques, unifiés par la période historique et culturelle et les limites géographiques, ne sont pas unis sur le plan de l'expression et des détails servant à créer l'image de la ville en question, puisque les attitudes mentales dont 
il s'agirait ne se rapportent pas uniquement à une époque donnée ni à une société en générale, mais comportent, en plus, des particularités individuelles du représentant de la culture regardante.

Aussi, l'image de Moscou serait-elle contrastée car, étant conditionnée par un stock limité d'images élémentaires très stables, elle ne coïncide pas avec les réalités du moment, comme le remarque Pageaux en parlant des visions stéréotypées (souvent clivées) des Espagnols, révélées dans les textes français (1989 : 135-136).

\section{3}

\section{LE MOSCOU DE SYLVAIN TESSON}

Tous les récits de voyage analysés combinent de nombreuses composantes communes, conçues pour créer l'image de la ville. Plus l'objectif du voyage est défini, plus l'image schématique de la ville est clair. Ainsi, l'idée du livre Berezina est apparue comme un geste de mémoire et de respect pour l'armée de Napoléon. On y trouve donc le retour artificiel au passé et la représentation de sa fusion avec le présent. Sylvain Tesson souhaite comprendre comment Moscou est apparue aux soldats de Napoléon et quelles impressions la ville a produit sur eux. En même temps, l'auteur voit un autre Moscou et l'image est créée en superposant le passé au présent, complétée par la fusion des contraires. Dans ce cas particulier, donc, l'image de la ville est construite au croisement de deux cultures françaises différentes car distancées dans le temps et sur le plan culturel, aussi bien que l'objet « regardé » est aussi considérablement changé à son tour.

Tesson construit l'image de Moscou sur un contraste :

1) Les Moscovites qu'il rencontre sont étonnamment multiformes : rassemblés et hommes d'affaires au travail, ils sont aussi de vrais villageois et parfois même ermites en dehors de la ville.

2) Les noms des rues ne coïncident pas en proportions avec leur taille, ce qui s'avère paradoxal.

La perception de Moscou semble ne pas avoir changé avec le temps. Ayant aveuglé Napoléon par sa beauté, Moscou surprend toujours le Français, non seulement avec ses étoiles en or et le Kremlin, mais aussi avec son architecture moderne, "grosse capitale de fer », (2015: 53). Tesson suppose que Moscou peut rendre amoureux d'elle et provoquer des émotions qui échappent au contrôle de la raison (le cas de Napoléon). L'écrivain compare le charme de la ville à la « beauté interdite » (2015:49), dont il convient de se méfier.

L'image de Moscou de Tesson est non seulement vivante, mais également active. L'auteur établit un parallèle avec la froideur d'une Moscovite, décisive et, comme le démontrent les événements d'il y a 200 ans, imprévisible et dangereuse. Dans le même temps, elle est attrayante et multiforme. 
Le récit de voyage de Dominique Fernandez est culturellement riche et se base sur deux visions venant d'« époques » différentes mais dans l'esprit d'un seul observateur (écrivain) : l'image de la capitale y est construite autour de sa propre vision de l'endroit, remplie de connaissances et d'émotions héritées de périodes succinctes et distancées dans le temps. Le chapitre sur Moscou est censé transmettre au lecteur un mélange d'émotions vécues par l'auteur lors d'une nouvelle rencontre avec la capitale russe qui a suivi celle d'il y a 20 ans. Cependant, l'auteur explique et justifie l'image controversée de Moscou qu'il s'est faite. Cette image est créée à l'intersection de l'éternel authentique et du moderne impersonnel. La sensation d'espace et de solitude, que Fernandez avait appréciée 20 ans plus tôt sur la Place Rouge, a été remplacée par la confusion et le regret à la vue d'un mélange impersonnel de "Champs-Élysées, de la Galerie Lafayette à la veille de Noël et de Las Vegas » (2012 : 25). Et, en dehors de cet étincellement, commence un immense espace d'une autre partie de la ville : pauvre, laide, mesquine, car seul le centre de Moscou, selon l'auteur, est affecté par l'invasion de l'esprit commercial. Et pourtant, l'auteur regrette cette déformation.

Cette soumission au négatif contraste avec la résilience de la ville. Fernandez mentionne que la partie périphérique de Moscou résiste jusqu'à présent, ajoutant que le reste de la Russie ne succombera jamais à cette influence.

\section{5}

\section{LE MOSCOU DE GÉRALDINE DUNBAR}

Le récit de Géraldine Dunbar est l'exemple remarquable des changements perpétuels des composantes de l'image de la ville en question. Pourrait-ce être, en quelque sorte, une particularité de l'objet de description ? Sensible aux différenciations minimes dans la ville, l'auteure remarque ces particularités des traits fugitifs de la ville tout en révélant les traits stables. Son récit est moins influencé par ce stock limité dont Pageaux parlait, elle se laisse saisir par les impressions qu'elle recueille à partir de l'espace examiné.

Pour Géraldine Dunbar, la visite à Moscou en 2010 n'est pas la première. La voyageuse amoureuse du pays, de sa culture et de son peuple possède une expérience et un bagage culturels - y compris la maîtrise de la langue russe favorisant la découverte de l'Autre - qui deviennent la base de l'image de la capitale russe dans ses récits de voyage publiés en 2016, récits très personnels, émotionnels et ouverts (2017). L'idée centrale autour de laquelle se construit l'image de Moscou est la métamorphose constante de la ville, très rapide (parfois en quatre mois de voyage en Extrême-Orient) et malheureusement dépourvue de sens. On remarque une tendance à la transformation constante - la ville suit la voie européenne en érigeant de hauts immeubles de verre pour le travail et la vie. Le 
désir de la voyageuse, ouverte aux contacts, de déceler l'attitude des locaux à l'égard des éléments architecturaux controversés de Moscou, se heurte à l'incapacité des citoyens à exprimer leur opinion. (N'est-ce pas un reflet des spécificités de la mentalité nationale : le manque de vision claire de soi dans la ville et, plus largement, de soi en tant que nation et, par conséquent, du désir d'autodétermination par le biais d'une transformation dans la recherche de l'identité propre aux Russes en général ? N'est-ce pas son but ultime ?)

Dunbar, connaissant bien l'histoire et la culture, regrette les métamorphoses de Moscou et leur dualisme du présent :

1. Moscou est fière de son patrimoine culturel, mais manque de respect envers celui-ci. Le centre historique regorge de restaurants et de boutiques, inaccessibles pour la plupart des habitants. Sur les façades des maisons marquées par la vie et le travail des artistes éminents, les plaques commémoratives voisinent avec les enseignes des magasins de lingerie.

2. Moscou a une grande histoire, mais la ville lutte contre son passé architectural, s'en débarrasse comme d'une maladie mortelle qui menace la vie, l'effaçant de la surface de la terre et de sa mémoire.

La personnification de Moscou augmente le degré d'empathie. Plus d'une fois, l'auteure appelle la ville «chère Moscou » $(2017: 60,313)$, la dotant d'un cœur en pleine mutation. Même en temps de paix, il n'y a pas de paix pour la ville : "Dans le cœeur fébrile de Moscou, partout des plaies » (2017 : 313). Pour tenter de trouver une comparaison appropriée pour la capitale, l'auteure revient à l'image d'un mannequin « que l'on habille et déshabille» (2017: 60). Dunbar écrit : "Ton style hétéroclite, libre, anarchique, souvent insolite. Qui es-tu? » (2017 : 61) Le mannequin inspire de l'admiration, mais ne possède et ne démontre malheureusement aucune volonté. Elle est vêtue de ce qui devrait être affiché et de ce qui devrait impressionner les autres. Ainsi, Dunbar explique l'hétérogénéité de l'image de Moscou, son hétérogénéité et non sa richesse polyvalente.

Les changements rapides dans l'aspect de la ville ressemblent à des défilés de mode, solennels, parfois pompeux, à la gaîté artificielle. L'auteure fait remarquer ce manque de naturel en évoquant l'incohérence dans le comportement et dans la vie des Moscovites. En se baladant dans le centre, Géraldine Dunbar interprète à son grand regret la publicité de la restauration rapide trouvée non loin de la Place Rouge : le Moscou authentique change, contrairement au Big Mac.

\section{6}

\section{LE MOSCOU DE HERVÉ BELLEC}

Le récit de voyage de Hervé Bellec Les Sirènes du Transsibérien (2008) démontre l'influence des stéréotypes sur la création de l'image d'un lieu. L'image de l'Autre, venant de la psychologie sociale, l'identité nationale et certaines particularités personnelles, serait 
donc un fruit de la catégorisation de toutes ces connaissances et sensations. L'auteur n'est pas toujours très tendre avec la ville de Moscou. Il s'y est trouvé un peu perdu ne connaissant pas la langue russe ; de plus, habitant habituellement en Bretagne, loin de Paris et tout près de la campagne, il se sentait effrayé par la capitale russe.

L'atmosphère de la ville est transmise à travers des faits historiques et des émotions personnelles de l'auteur. Moscou s'y présente comme modèle à imiter. Pourtant, cette image n'est pas celle d'un mannequin, mais d'un modèle stéréotypé permettant de créer une ville similaire. La comparaison avec un stéréotype, en parallèle avec celle d'un mannequin, n'est pas un compliment pour la ville. L'auteur considère toutefois que ces caractéristiques sont en quelque sorte positives : Moscou est l'une des villes les plus logiques et les plus faciles à comprendre.

Moscou, c'est la concentration de tout ce qu'il y a dans le pays et de tout ce à quoi le pays aspire. Mais en même temps, Moscou est le modèle du fossé entre les composantes de la nation, et c'est un fossé infranchissable. Il n'y a pas de juste milieu en Russie, il y a des extrêmes, et pour l'auteur, ces extrêmes sont incarnés dans l'architecture de la capitale, des voitures, des scènes dans les églises. Cette idée est confirmée par le choix des personnages décrits.

Les extrêmes, comme l'auteur le voit, changent souvent de lieu, provoquant des contradictions relevées par de nombreux auteurs : le dogme devient folklore (Arbat), la puissance militaire du pays n'est pas en corrélation avec l'état des transports en commun, l'image de la ville de Moscou authentique est nivelée par de nombreux cafés sans visage. Bellec relève avec surprise que le métro de Moscou est l'élément le plus solide de l'image de la ville : la réserve des chefs-d'œuvre, le métro reste stable face au temps et aux hooligans.

En raison de son métier, Bellec connaît bien l'histoire de la Russie. Trois jours passés dans la capitale représentent donc pour lui une visualisation constante de ses savoir sur le passé, tout en détruisant ou en confirmant les idées stéréotypées sur la ville moderne de Moscou. Bien que ce soit un modèle, celui-ci est compréhensible mais rend sceptique, c'est un modèle dont l'auteur ne cesse de se méfier (passants, passagers, transports). Le point de vue d'un Européen, ce qui détermine la perception de la ville russe, ne permet pas à Bellec de l'accepter et de percevoir ses qualités positives. Craignant les contacts avec des étrangers (parmi lesquels, selon ses informations préliminaires, on rencontrerait de nombreux représentants de la mafia et des travailleurs ordinaires, absolument indifférents à la grandeur de leur pays et de leur culture), l'auteur parle avec joie et apaisement à une connaissance française rencontrée à Moscou, "quelqu'un de normal, civilisée et raisonnable » (2008: 81, 93). L'écrivain est trop prudent, il ne communique avec aucun moscovite, à l'exception de sa guide qui ne suscite d'ailleurs pas l'intérêt de l'écrivain - " ponctuelle, main particulièrement ferme ; une vraie Russe " (2008 : 50, 52, 70). L'image de Moscou par Bellec exclut les Moscovites en tant qu'élément constitutif, pour lui ce n'est qu'une «foule, gens, passagers, silhouettes » $(2008: 83,96,57)$. Cependant, 
certaines caractéristiques de cette foule, telles que l'inattention aux autres, le manque de sourire, le fatalisme, l'équanimité, l'imperturbabilité amènent l'auteur à définir Moscou comme une « ville monstrueuse » (2008:111).

Moscou est donc un modèle stéréotypé, une ville d'histoire, une ville cible de tout le pays, une ville d'indifférence. L'étrangeté de l'image de la ville est accompagnée et renforcée par le choix des mots et expressions russes enregistrés par translittération, dans de rares cas, sans traduction. L'auteur met donc d'avantage l'accent sur le contenu des stéréotypes les plus courants sur la Russie, ce qui est censé faciliter la perception assez exotique de la ville pour un Européen. Bellec essaye de traduire ses émotions en les mêlant à ses connaissances historiques et en jouant avec les habituels clichés qu'un Français peut porter sur un pays étranger.

\section{CONCLUSION}

Les récits de voyage présentés sont variés (style, émotions, composition), mais leur valeur commune est de nature culturelle : certains révèlent au lecteur une base historique et culturelle solide, d'autres sont remplis de traces émotionnelles sur le plan de la ville ou fournissent des informations pratiques. La particularité des récits de voyage en général et ceux analysés en particulier réside dans l'apport culturologique : les accents (linguistiques, culturels, sociaux) mis par les écrivains reflètent la mentalité nationale et les attitudes culturelles des auteurs.

Le point de vue français sur Moscou est non seulement une représentation de l'image de la ville russe, mais également une traduction de l'image typique d'une capitale, inscrite dans la conscience nationale française dans son ensemble et en particulier dans la conscience culturelle individuelle de chacun des écrivains. Ces exigences implicites sont exprimées sous forme d'accents émotionnels de regret, de ressentiment, d'appréhension, de surprise, d'admiration, communiqués par les auteurs.

Dans certains récits de voyage, on remarque une tendance à préserver le patrimoine culturel, la mémoire historique et l'identité nationale. D'où la surprise et, parfois, la déception face aux métamorphoses rapides de Moscou.

Dans presque tous les récits de voyage, l'image de Moscou est dualiste, et globalement, ces images sont contradictoires. Toujours caractérisée par un élément de métamorphose, Moscou se voit tantôt comme une ville active et décisive, tantôt comme un objet de manipulations et un espace pour la réalisation des idées de l'extérieur. Chacun des auteurs a sa vision personnelle et se donne la liberté de la traduire en mot, c'est tout l'intérêt des récits des voyages. En outre, la réflexion sur les stéréotypes que se forge l'Autre se révèle fructueuse pour étudier l'image de soi car c'est souvent l'opposé qui attire l'attention en premier lieu. 


\section{BIBLIOGRAPHIE}

BECK, Philippe (2010) Imagologie, psychologie sociale et psychologie cognitive. H. Roland et S. Vanasten (dir.), Les nouvelles voies du comparatisme. Gent : Academia presse, 57-69. 18 octobre 2019. https://www.academia.edu/5030154/ Imagologie_psychologie_sociale_et_psychologie_cognitive.

BELLEC, Hervé (2008) Les Sirènes du Transsibérien. Paris : Géorama.

DUNBAR, Géraldine (2017 [2016]) Seule sur le Transsibérien. Paris : Transboréal.

FERNANDEZ, Dominique (2012) Transsibérien. Paris : Grasset.

LIPPMANN, Walter (1922) Public Opinion. 18 octobre 2019. http://www.gutenberg. org/cache/epub/6456/pg6456-images.html.

MOUREAU, François (2016) Diderot, un philosophe en voyage. I. Zatorska (dir.), Rousseau et Diderot, traduire, interpréter, connaître. Varsovie : Wydawnictwa Uniwersytetu Warszawskiego, 65-84. 18 novembre 2019. https://www.academia. edu/29430948/_Diderot_un_philosophe_en_voyage_Rousseau_et_Diderot_traduire_interpr\%C3\%A9ter_conna\%C3\%AEtre_Izabella_Zatorska_dir._Warszawa Wydawnictwa_Uniwersytetu_Warszawskiego_2016_p._65-84.

PAGEAUX, Daniel-Henri (1989) De l'imagerie culturelle à l'imaginaire. P. Brunel et Y. Chevrel (dir.), Précis de littérature comparée. Paris : PUF, 133-162.

PAGEAUX, Daniel-Henri (1995) Recherche sur l'imagologie : de l'Histoire culturelle à la Poétique. Revista de Filología Francesa 8, 135-160. 18 octobre 2019. https:// www.academia.edu/24783312/Recherche_sur_1imagologie_de_1Histoire_culturelle_\%C3\%A1_la_Po\%C3\%A9tique.

TESSON, Sylvain (2016) Berezina. Paris : Folio.

\section{POVZETEK}

\section{Podoba Moskve v potopisnih pripovedih sodobnih francoskih pisateljev}

Potopisna pripoved je priljubljena zvrst v sodobni književnosti. Njeni začetki segajo v 5. stoletje pred našim štetjem, ko je bila njena vloga predvsem utilitaristična. Osrednje mesto v potopisni pripovedi ima opis (narave, mest, prebivalcev, simbolov, osebnih vtisov in asociacij), medtem ko ima dogajanje stransko oziroma podporno vlogo. Potopisna pripoved je v zadnjih tridesetih letih v Rusiji in izven nje postala zelo priljubljena zvrst, kljub temu da je potovanje postalo dostopnejše vsem. Prav dostopnost potovanja je spremenila zasnovo potopisnih pripovedi, s tem pa je vplivala tudi na njihovo vsebino. V prispevku analiziramo podobo Moskve $\mathrm{v}$ potopisih francoskih avtorjev, objavljenih med letoma 2008 in 2016, ki večinoma opisujejo potovanja po Sibiriji. 
Posamezna dela se razlikujejo glede na način posredovanja avtorjevega dojemanja sveta, glede na pogled na mesto in na interpretacijo videnega. To raznolikost je mogoče pripisati različnim dejavnikom, kot so avtorjeva narodnost, poklic, življenjske izkušnje, spol, namen potovanja, pa tudi predhodne izkušnje s kulturo države in znanjem ruskega jezika. Podobo Moskve v preučevanih delih tako oblikujejo zgodovinska dejstva, spomini, primerjava s podobami Moskve v preteklosti, toponimija, zgradba mesta, meščani in srečanja z njimi ter primerjava predsodkov in resničnosti. Dojemanje tuje prestolnice pa na koncu razkrije avtorjevo podzavestno dojemanje naroda in sebe.

Ključne besede: potopisna pripoved, podoba mesta, podzavestno dojemanje naroda, stereotip, imagologija

\section{ABSTRACT}

\section{The image of Moscow in travel narratives of contemporary French writers}

Born in the fifth century BC for utilitarian purposes, the travel narrative is a common genre in modern literature. Description (nature, cities, inhabitants, symbols, personal impressions and associations) is the main and immutable component of these works, while action also plays a supporting role. The genre of travel narrative has gained particular popularity over the last thirty years, not only in Russia but also abroad, despite the fact that traveling has become more accessible to everyone. This situation has changed the concept of such works, and consequently their content. The image of Moscow is analysed from the travelogues of French authors published from 2008 to 2016, most of which describe the trans-Siberian voyage.

The means of delivering of the author's vision, their perception of the city and the interpretation of what they have seen, are diverse and conditioned by different factors: nation, profession, biography, gender, travel intentions, as well as previous cultural experience and mastery of the Russian language. Thus, the image of Moscow is revealed in the indicated works through historical facts, memories and comparisons with the past image of Moscow, toponymy, the morphology of the city, its people and meetings with them, and also through a comparison of prejudices and what is real. It is ultimately the national and personal unconscious that emerges in the sense of the foreign capital.

Keywords: travel account, image of the city, national unconscious, stereotype, imagology

\section{RÉSUMÉ}

Né au $\mathrm{V}^{\mathrm{e}}$ siècle avant J.-C. à des fins utilitaires, le récit de voyage est un genre répandu dans la littérature moderne. La description (nature, ville, habitants, symboles, impressions personnelles et 
associations) est le composant principal et immuable de ces œuvres. L'action joue également un rôle de soutien. Le genre du récit de voyage a acquis une popularité particulière au cours des trente dernières années, non seulement en Russie mais aussi à l'étranger, malgré le fait que les voyages sont devenus plus accessibles à tout le monde. Cet état de fait a corrigé le concept d'œuvres et, en conséquence, leur contenu. L'image de Moscou est analysée d'après les récits de voyages d'auteurs français publiés de 2008 à 2016, dont la plupart décrivent le voyage transsibérien.

Les moyens de transmission de la vision de l'auteur, de sa perception de la ville et de l'interprétation de ce qu'il a vu, sont divers et sont conditionnés par des facteurs différents : national, professionnel, biographique, celui de genre, des intentions de voyage, ainsi que de son expérience culturelle vécue auparavant et sa maîtrise de la langue russe. Ainsi, l'image de Moscou est révélée dans les œuvres indiquées à travers des faits historiques, des souvenirs et des comparaisons avec l'image passée de Moscou, la toponymie, la morphologie de la ville, les gens et les rencontres avec eux, à travers une comparaison du réel et des préjugés. C'est finalement l'inconscient national et personnel qui ressort dans l'acception de la capitale étrangère.

Mots-clés : récit de voyage, image de la ville, inconscient national, stéréotype, imagologie 